\title{
Colorless devices and reception techniques for polarization multiplexed communications
}

\author{
Robert Halir, ${ }^{1}$ Pedro Reyes-Iglesias, ${ }^{1}$ Carlos Alonso-Ramos, ${ }^{2}$ Darío Sarmiento-Merenguel, ${ }^{1}$ Gonzalo \\ Wangüemert-Pérez, ${ }^{\mathbf{1}}$ Pavel Cheben, ${ }^{3}$ Íñigo Molina-Fernández, ${ }^{\mathbf{1}}$ Alejandro Ortega-Moñux ${ }^{\mathbf{1}}$ \\ ${ }^{1}$ Universidad de Málaga, ETSI Telecomunicación, Campus de Teatinos s/n, 29071 Málaga, Spain \\ ${ }^{2}$ Institut d'Electronique Fondamentale, Unive. Paris-Sud CNRS-UMR8622, Bat. 220 F-91405 ORSAY, France \\ ${ }^{3}$ National Research Council of Canada, Ottawa, Ontario, K1A OR6, Canada \\ e-mail: robert.halir@ic.uma.es
}

\begin{abstract}
Future optical networks call for flexible, high performance and low cost coherent optical receivers. We present here several advances towards such receivers, including integrated optical couplers with ultra-broad bandwidth, as well as novel reception techniques and architectures that will enable high performance coherent reception without filtering and polarization splitting elements.
\end{abstract}

Keywords: coherent receivers, colorless operation, multimode interference couplers, polarization splitters, OSNR penalty, receiver architectures

\section{INTRODUCTION}

Optical transmission networks are evolving from classical long-haul scenarios towards flexible, high speed optical access networks [1]. These networks call for optical components that offer high performance and low cost while being suitable for diverse network scenarios. This has spurred interest in coherent receivers that can provide colorless operation in a broad spectral range and can work with a wide range of input powers, i.e. offer high dynamic range [2]. In order to achieve rugged devices and reduced costs, such receivers would ideally be fabricated as monolithically integrated chips. As shown in Fig. 1, this requires, among other factors, the integration of polarization beam splitters (for polarization multiplexing) and high performance $90^{\circ}$ hybrids combined with high speed photodiodes (for optical down-conversion); trans-impedance amplifiers are typically wire-bonded to the optical chip. While important advances have been achieved in both aspects [3], [4], it is still challenging to achieve high-yield (and thus economically viable) solutions for $90^{\circ}$ hybrids simultaneously covering the $\mathrm{C}+\mathrm{L}$ bands $(1530 \mathrm{~nm}-1625 \mathrm{~nm})$ or fully passive on-chip polarization splitters.

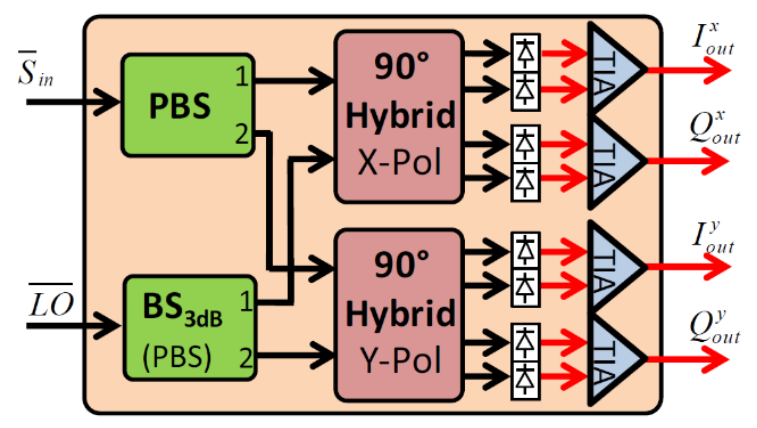

Figure 1. Schematic of a polarization multiplexed coherent optical receiver comprising polarization splitters (PBS), $90^{\circ}$ hybrids (typically implemented with MMIs), photodiodes and trans-impedance amplifiers [8]. In the signal path, the polarization beam splitter $(P B S)$ is mandatory. In the LO path, two alternatives can be used: a polarization insensitive power divider $(P B S+B S$ scheme) or a polarization beam splitter $(P B S+P B S$ scheme).

Here, we review some of our recent advances, both on the device and system level, towards flexible coherent optical receivers. Specifically, in sections 2 and 3 we will discuss the design of colorless multimode interference couplers and a calibration technique to compensate for imperfect $90^{\circ}$ hybrids. Regarding polarization management, we introduce an analytic expression to evaluate the OSNR penalty of imperfect polarization splitters (section 4), and propose a receiver architecture that operates without any polarization splitting elements (section 5). Conclusions are drawn in section 6.

\section{COLORLESS MULTIMODE INTERFERENCE COUPLERS}

In most state-of-the-art receivers, $90^{\circ}$ hybrids are implemented with multimode interference couplers (MMIs); more specifically with 2x4 MMIs. While MMIs are relatively broadband devices, it is well known that their bandwidth scales inversely with the number of input and output ports. In fact, even without taking into account the effect of fabrication tolerances, a 2x4 MMI only complies with OIF (Optical Internetworking Forum) 
specifications in a band slightly larger than the C-band [5]. The implementation of high-performance receivers with much broader bandwidths (covering, for instance the $\mathrm{S}, \mathrm{C}$ and $\mathrm{L}$ bands simultaneously) thus requires a different approach to MMI design. In [6], [7] we proposed the use of sub-wavelength structures to significantly enhance the bandwidth MMI devices. Such segmented devices, shown in Fig. 2, achieve an approximately fivefold bandwidth enhancement compared to conventional MMI. In fact, if successfully implemented, such a device could cover all optical communication bands.

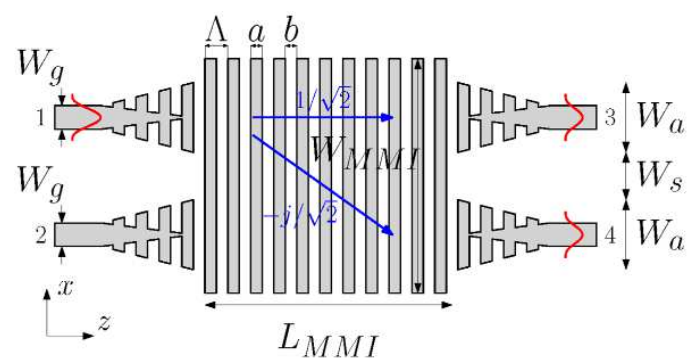

Figure 2. Geometry of an ultra-broadband, sub-wavelength grating based MMI.

However, the implementation is rather challenging, as such a device poses stringent tolerance requirements, which are difficult to achieve with current fabrication techniques. As high yield is an important factor for commercially viable receivers, other alternatives to achieve broad-band colorless operation are needed.

\section{RECEIVER CALIBRATION FOR COLORLESS OPERATION}

When a coherent receiver is operated without pre-filtering the desired channel (i.e. colorless operation), its performance is limited by an additive interfering baseband signal, arising from the self-beating of the adjacent channels [8], [9]. This interference is proportional to the power of the received signal (thereby limiting dynamic range), but is suppressed if the $90^{\circ}$ hybrids exhibit a high common mode rejection ratio (CMRR>20dB). However, implementing such high-performance hybrids with relaxed fabrication tolerances, while at the same time achieving broad bandwidth, is very challenging. We therefore proposed a calibration technique that enhances the "effective" CMRR of the hybrids, thereby suppressing the interfering signal and enabling colorless operation over a broad bandwidth [8].

a)

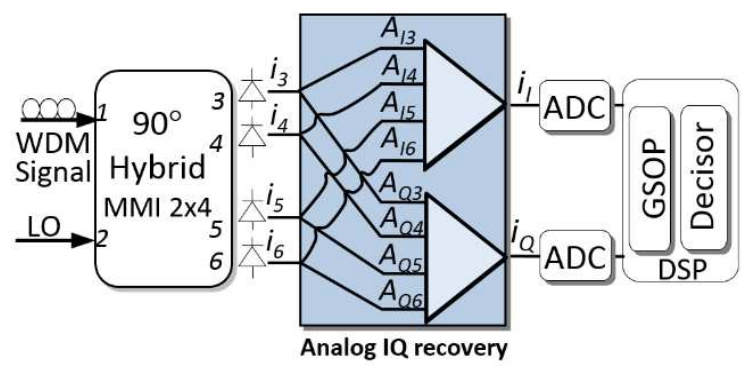

b)

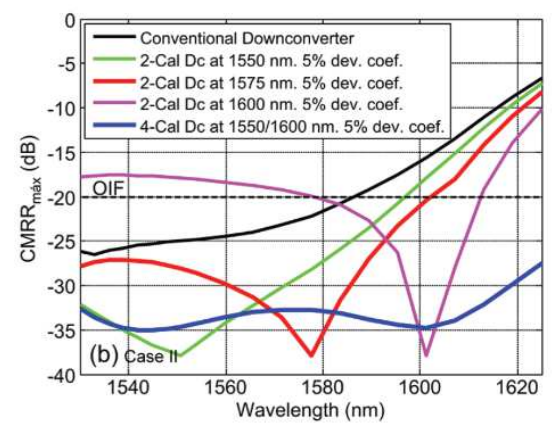

Figure 3. (a) An architecture for colorless coherent detection based on analogue calibration of the $90^{\circ}$ hybrid.

(b) Equivalent Common Mode Rejection Ratio of the $90^{\circ}$ hybrid with and without analogue calibration [8].

The architecture is shown in Fig. 3(a); instead of directly subtracting the currents from the paired photodiodes as in a conventional receiver (Fig. 1), analogue adders, with weights $A_{I Q i}$, feed a linear combination of all photocurrents into the analogue digital converters (ADC). The weights are determined once by receiving a small number of known symbols and then remain static. Figure 3(b) shows the effective CMRR of a $90^{\circ}$ hybrid subjected to realistic fabrication tolerances without any calibration ("Conventional Downconverter") and after calibration, taking into account a $5 \%$ deviation of the weights $A_{I Q i}$ ("4-Cal Dc"). It is apparent that the CMRR is improved between $10 \mathrm{~dB}$ and $20 \mathrm{~dB}$ over the full $\mathrm{C}+\mathrm{L}$ bands. The predicted dynamic range for this calibrated device exceeds $20 \mathrm{~dB}$.

\section{AN ANALYTICAL METRIC FOR THE IMPACT OF NON-IDEAL POLARIZATION SPLITTERS}

The monolithic integration of passive polarization splitting elements is another major challenge for receiver design. Off-chip polarization beam splitters can offer very high polarization extinction ratios (PER 20dB), which are, however, very difficult to achieve with passive on-chip devices that have reasonable fabrication tolerances. In [10] we derived, for the first time, an analytical expression to assess the actual optical signal-to-noise ratio penalty that arises from using imperfect polarization splitters ( $\triangle$ OSNR). Such an expression is extremely useful, as it enables 
the definition of clear specification "interfaces" for hardware design, without having to simulate the full receiver chain including the DSP algorithms. Specifically, let $\mathbf{M}$ be the Jones Matrix describing the downconverter in Fig. 1. If $\left\|\mathbf{M}^{-1}\right\|^{2}$ is the square Frobenius norm of the inverse matrix, i.e. the sum of the squared modulus of its elements, the OSNR penalty is directly given by:

$$
\Delta \text { OSNR }[\mathrm{dB}]=10 \log \left(\left\|\mathbf{M}^{-1}\right\|^{2} / 2\right) .
$$

Figure 4(a) compares the OSNR penalty due to imperfect polarization splitters as obtained through simulation of the full receiver chain (including digital orthogonalization and polarization demultiplexing algorithms - GSOP and CMA) and equation (1). It is clear that a system where both the local laser and the signal are fed through polarization splitters (PBS+PBS scheme in Fig. 1) offers a 3dB advantage over implementations where the local laser is divided simply by a beam splitter (PBS+BS scheme in Fig. 1). Furthermore, it is remarkable that even with a relatively poor polarization extinction ratio of about $10 \mathrm{~dB}$ (which can be achieved with monolithically integrated polarization splitters) the OSNR penalty is only $\sim 1 \mathrm{~dB}$ with the PBS+PBS scheme.

a)

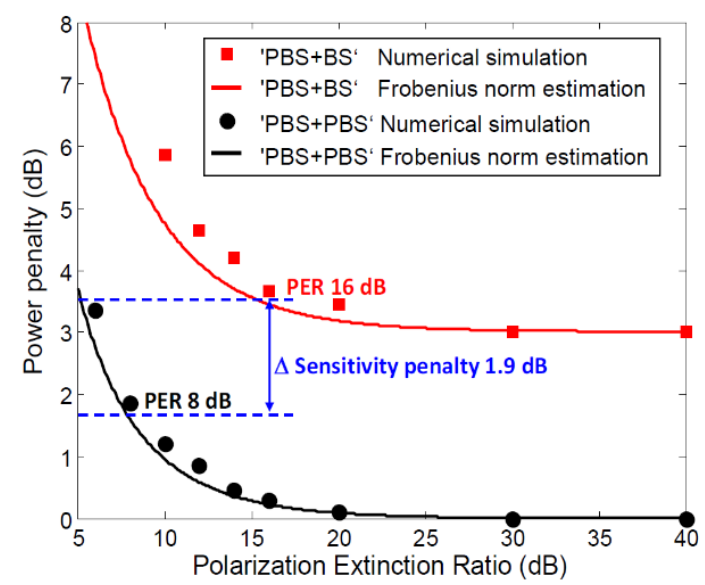

b)

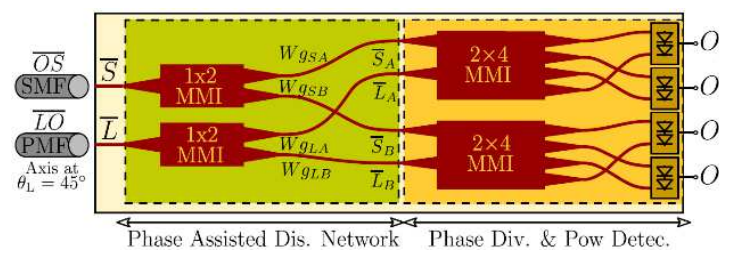

Figure 4. (a) OSNR penalty due to imperfect polarization beam splitters [10] (b) An architecture for polarization-beam-splitter-less coherent detection based on birefringence engineered waveguides [11].

\section{A POL-MUX RECEIVER ARCHITECTURE WITHOUT POLARIZATION SPLITTERS}

An interesting alternative to the aforementioned architecture with integrated polarization splitters is obviating such elements altogether. This is possible with the receiver structure shown in Fig. 4(b), where polarization demultiplexing is implemented by a combination of birefringence engineered waveguides on the chip (phase assisted distribution network), and the local laser input fiber being aligned with $45^{\circ}$ tilt in its polarization axis respect to the chip [11]. In terms of fabrication tolerances such a device is far more robust than implementations relying on integrated polarization splitters. Regarding OSNR, this architecture offers the same performance as a conventional receiver with a polarization splitter only for the signal input (PBS+BS scheme in Fig. 1).

\section{CONCLUSIONS}

The implementation of commercially viable, fully integrated coherent receivers that can offer colorless operation over a broad spectral range still faces a number of challenges. In this paper we have presented several techniques that address these challenges. Regarding the performance of the integrated $90^{\circ}$ hybrids, we have introduced multimode interference couplers that can potentially cover several full communication bands, and a hardware calibration procedure that can compensate for imperfect hybrids, improving their effective CMRR by at least an order of magnitude. We have furthermore established an analytical metric to assess the impact of imperfect integrated polarization splitters, revealing that a moderate polarization extinction ratio of $10 \mathrm{~dB}$ introduces an OSNR penalty of only $\sim 1 \mathrm{~dB}$, and have suggested an architecture that eliminates the need for polarization splitting devices altogether.

\section{ACKNOWLEDGEMENTS}

This work was supported by the Spanish Ministry of Science under project TEC2013-46917-C2-1-R, and the Universidad de Málaga - Campus de Excelencia Internacional Andalucía Tech. 


\section{REFERENCES}

[1] S. J. Savory: Digital Coherent Optical Access Networks, IEEE Photonics Conference (IPC), pp. 125-126, 2013.

[2] M. Morsy-Osman et al.: Analytical and experimental performance evaluation of an integrated Si-photonic balanced coherent receiver in a colorless scenario, Opt. Express, vol. 22, pp. 5693-5730, 2014

[3] H. Mardoyan, O. Bertran Pardo, P. Jennevé, G. de Valicourt, M. A. Mestre, S. Bigo, C. Kazmierski, N. Chimot, A. Steffan, J. Honecker, R. Zhang, P. Runge, A. Richter, C. Arellano, A. Ortega-Moñux, and I. Molina Fernandez: PIC-to-PIC experiment at $130 \mathrm{~Gb} / \mathrm{s}$ Based on a Monolithic Transmitter Using Switching of Prefixed Optical Phases and a Monolithic Coherent Receiver, in Optical Fiber Communication Conference 2014: Postdeadline Papers, paper Th5C.2, 2014

[4] D. Pérez-Galacho, R. Zhang, A. Ortega-Moñux, R. Halir, C. Alonso-Ramos, P. Runge, K. Janiak, G. Zhou, H.G. Bach, A.G. Steffan, and I. Molina-Fernández: Integrated Polarization Beam Splitter for 100/400 GE Polarization Multiplexed Coherent Optical Communications, J. Lightwave Technol., vol. 32, pp. 361-368 2014

[5] Implementation Agreement for Integrated Dual Polarization Intradyne Coherent Receivers, Optical Internetworking Forum, Available: http://www.oiforum.com/public/documents/OIF_DPC_RX-01.2.pdf

[6] A. Maese-Novo, R. Halir, S. Romero-García, D. Pérez-Galacho, L. Zavargo-Peche, A. Ortega-Moñux, I. Molina-Fernández, J. G. Wanguemert-Pérez, and P. Cheben: Wavelength-Independent Multimode Interference Coupler, Opt. Express, vol. 21, pp. 7033-7040, 2013

[7] R. Halir, P. Bock, P. Cheben, A. Ortega-Moñux, C. Alonso-Ramos, J. Schmid, J. Lapointe, D.-X. Xu, J. G. Wangüemert-Pérez, Í. Molina-Fernández and S. Janz: Waveguide sub-wavelength structures: a review of principles and applications, Laser Photon. Rev., vol. 9, pp. 25-49, 2015

[8] P.J. Reyes-Iglesias, A. Ortega-Moñux, I. Molina-Fernández: Calibrated Monolithically Integrated 90 Downconverter for Colorless Operation in the C+L Band, IEEE Photon. J., vol.7, p. 7200710, 2015

[9] P.J. Reyes-Iglesias, A. Ortega-Moñux, I. Molina-Fernández: Colorless monolithically integrated 120 downconverter, Opt. Express, vol. 21, pp. 23 048-23 057, 2013

[10] P. J. Reyes-Iglesias, I. Molina-Fernández, and A. Ortega-Moñux: Sensitivity penalty induced by non-ideal dual polarization downconverter in digital coherent receivers, Opt. Express, vol. 23, pp. 12784-12794, 2015

[11] C. Alonso-Ramos, P. J. Reyes-Iglesias, A. Ortega-Moñux, D. Pérez-Galacho, R. Halir, and I. MolinaFernández: Polarization-beam-splitter-less integrated dual-polarization coherent receiver, Opt. Lett., vol. 39, pp. 4400-4403, 2014 